\title{
Study on Blend Irregularity Caused by Drafting.
}

\author{
Part 1: Theoretical and Experimental Analysis. \\ By Shigeji Yamashita, ${ }^{*}$ Sueo Kawabata, ${ }^{* *}$ and Hiromichi Kawai, ** Members, TMSJ \\ *Textile Laboratory, Toyo Rayon Co., Ltd., Otsu, **Faculty of Engineering, Kyoto University, Kyoto. \\ Based on the Journal of the Textile Machinery Society of Japan, Transactions, Vol. 20, No. 6, T62-63(1967-5)
}

\section{Introduction}

With the recent development of new textile products, many kinds of blended spun yarns have been produced.

In order to design new kinds of textile products using blended yarns it is important to understand the structures and properties of blended yarns such as thickness irregularity and blend ratio irregularity.

The blend irregularity has been studied by many workers $[1-7]$. The subjects such as the effects of doubling on the blend irregularity, the fiber distribution in the yarn cross section, have been discussed. Most of those reports were concerned with the static analysis of blended yarn structure and not with the dynamic analysis of blend irregularity caused by drafting. In the measurement of the blend ratio in the cross sections along a yarn, two types of blend ratio irregularity are observed. One is longer period irregularity and the other is shorter period one. It is easier to analyze the cause of longer period irregularity than that of shorter period one. Because the former is caused only by unsufficient mixing of blend components in the preparatory process of spinning, the latter is more complicated and the mechanism remains unexplained yet.

This paper is concerned with the shorter period blend ratio irregularity caused by drafting as follows:

(1) A model for the mechanism of the occurrence of blend ratio irregularity during the drafting when two components are blended is presented theoretically. In this model, blend ratio irregularity depends on the following factors:

Degree of capacity of the drafting system.

Controlling floating fiber of each component.

Degree of mutual interference between different fiber components during drafting.

(2) In order to ascertain the propriety of the model, experimental studies are made. In this experiment two kinds of fibers having different properties are blended, and the floating fibers of one component are more difficult to control by drafting system than those of the other. Two blending methods are used, blending of the two component is achieved either in blowing and blending machine or in the fine spinning process. Sample yarns were produced under different controls of floating fibers.

The thickness irregularity is measured with an electro-capacity type evenness tester. After one component of the fibers is dissolved in a solvent keeping the yarn form as it is. The thickness irregularity of the remaining component is measured. By this measurement, the thickness irregularity of these two components along the yarn axis are obtained and the blend ratio irregularity are calculated.

These experimental results are discussed using the theoretical model.

\section{Theoretical}

2-1. Mechanism of Occurrence of Thickness Irregularity during Drafting

It is generally believed that thickness irregularity occurring during drafting is due to floating fibers in the drafting zone behaving irregularly in the course of time. Theoretical and experimental research on draft has been done a lot and this work has started from the theory of Fujino and Kawabata. The summary of this theory is as follows (for the benefit of our work):

(1) In the ordinary drafting process, most floating fibers are considered to be subjected to steady drafting according to a given distribution of moving boundary. These stable floating fibers are temporarily excluded from the subject of study.

(2) They assume that mechanically unstable floating fibers affected by fibers nipped with front-rolls are wrongfully accelerated before they reach the normal speed 
change point (This is not necessarily a given location and has a certain distribution.) and that the number of accelerated unstable floating fibers is proportional to the increasing velocity of the mass of front-nipped fibers. Also it is assumed that fibers are instantly accelerated from the surface speed of the back rolls to that of the front ones at the speed change point.

(3) The probability that unstable floating fibers exist becomes greater as they come near the front nip point. Therefore, they assume that the probability distribution function of the speed change point of them is given as the exponential function of distance $(x)$ from the front nip point to the direction of the back rolls.

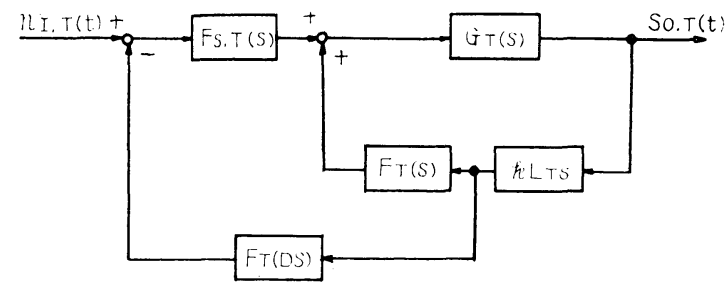

Fig. 1 Analogue block diagram of drafting process

The lapse of time of this drafting process is expressed as an analogue block diagram in Fig. 1 in S-domain Laplace transformed, where ; $N_{l}, T(t)=$ density function of fiber leading ends of supplied sliver in time-domain, $G t(S)=$ transfer function converting density function of fiber leading ends of supplied sliver in time domain into sliver thickness, $k=$ constnat of the proportion when unstable floating fibers are supposed to be accelerated in proportion to the increasing velocity of front nipped fibers, $L t=$ fiber length expressed in time domain, $F t(S)=$ probability distribution function of the velocity change point Laplace transformed of unstable floating fiber groups expressed in time domain, $F s, T(S)=$ probability distribution function of the velocity change point of stable, floating fibers expressed in time domain, taking the Laplace transformation, $F t(D S)=$ element in which the quantity of density function of fiber leading ends reduced in proportion to the number of ends of accelerated, unstable, floating fibers expressed in time domain, is deducted from supplied sliver $(D=$ draft ratio), and So, $T(t)=$ thickness of drafted sliver expressed in time domain.

And in the case of square cut fibers $(G \mathrm{~T}(S))$,

$$
G_{T}(S)=\frac{1}{S L_{T}}\left(1-l^{-S L T}\right)
$$

Probability distribution function of the velocity change point $(f(x))$ of unstable floating fibers is expressed by the following exponential function;

$$
f(X)=\frac{10}{L} e^{-\frac{10}{L} x}
$$

If the velocity change point distribution of normal floating fibers are assumed to be an approximate point;

$$
F_{s, T}(S)=1
$$

2-2. Mechanism of Occurrence of Blend Ratio Irregularity during Drafting

In order to clarify blend ratio irregularity caused by drafting it is essential that thickness irregularity of each component of blended sliver be studied. We assume that the thickness of blended yarn composed of " $A$ " and " $B$ " types is measured by separating " $A$ " from " $B$ " (See Fig. $2)$. Let thickness of the blended yarn in the cross section of coordinate $(x)$ along the yarn axis be $\mathrm{S}(x)$ and those of " $A$ " and "B" be $\mathrm{S}_{\mathrm{A}}(x)$ and $\mathrm{S}_{\mathrm{B}}(x)$ respectively.

$$
S(x)=S_{A}(x)+S_{B}(x)
$$

Blend ratio $(P(x))$ on coordinate $(x)$ shall be defined by the following formulas;

Thus,

$$
\begin{aligned}
& P_{A}(x)=\frac{S_{A}(x)}{S(x)} \times 100 \\
& P_{B}(x)=\frac{S_{B}(x)}{S(x)} \times 100
\end{aligned}
$$

$$
P_{A}(x)=\left\{\frac{S(x)-S_{B}(x)}{S(x)} ; \times 100=100-P_{B}(x)\right.
$$
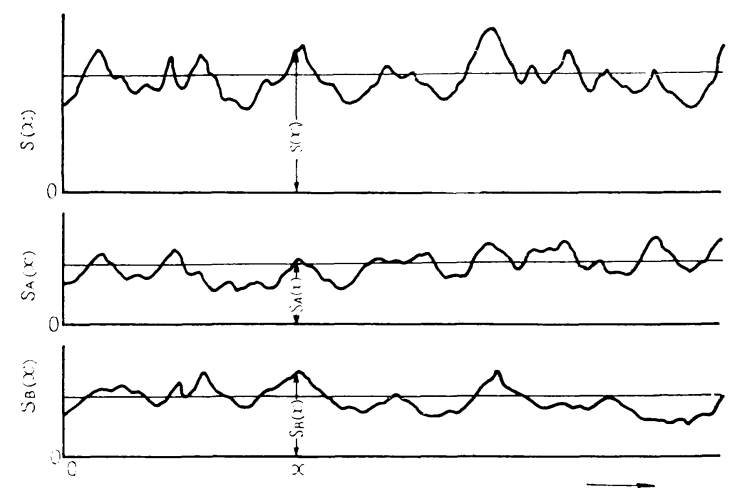

Fig. 2 An example of thickness irregularity curves of blended yarn and its two components 

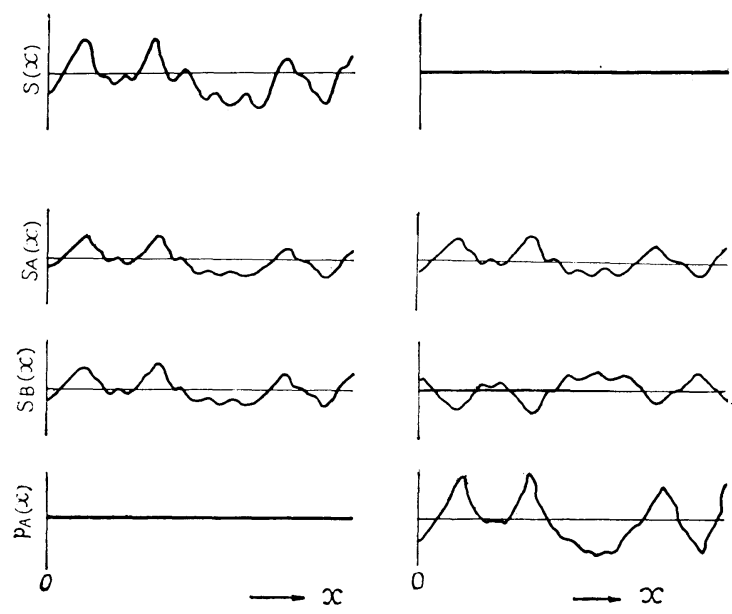

in the case of $r=1$

in the case of $r=-1.0$ where $r$ is correlation coefficient between $S_{A}(x)$ and $S_{B}(x)$

Fig. 3 Relation between thickness irregularity and blend ratio irregularity

Then, we will consider correlation between thickness of "A" component $(\mathrm{SA}(x))$ and that of "B" component $(S B(x))$. Let us see the following simple example; As seen in Fig. 3, if the average blend ratio is $50 \%$, the thickness irregularity of " $A$ " and " $B$ " is equal, and the correlation coefficient is 1.0 between thicknesses of each component at any given location of the blended sliver, blend ratio irregularity will not occur. If the correlation coefficient between the thickness of the two components is smaller than 1.0 it will appear. Further, if the coefficient becomes -1.0 , blend ratio irregularity will reach the maximum despite of the disappearance of thickness irregularity of the blended yarn (See Fig. 6).

As mentioned above, blend ratio irregularity depends upon the thickness irregularity of each component fiber and the correlation between the thickness irregularities of both the fibers. Let us see floating fiber motion in blended sliver under drafting. It is conceivable that at the front nip point, nipped " $A$ " and " $B$ " fibers affect the acceleration of unstable floating " $A$ " and " $B$ " fibers.

Therefore, we will simulate the following two fundamental structures for the drafting process of a blended sliver. (1) Two types of fibers are located separately in each cross section of blended sliver.

In this case, " $A$ " and " $B$ " are located separately as seen in Fig. 4 and so they do not affect each other. They are drafted independently - that is, the unstable, floating fibers of " $A$ " and " $B$ " are accelerated in proportion to increasing velocity of front nipped fibers " $A$ " and " $B$ ".

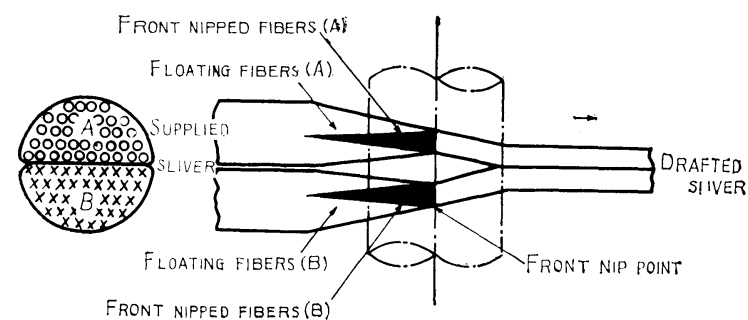

Fig. 4 Fibers near front rollers when two kinds of fibers are located separately in each cross section of the blended sliver

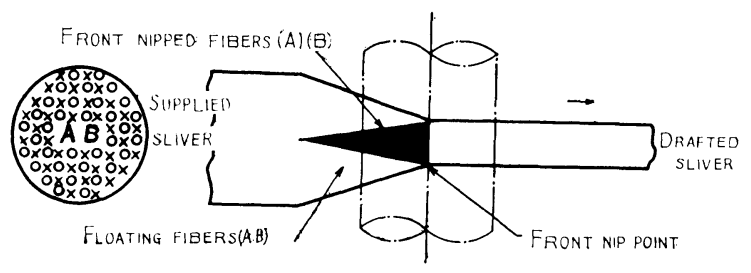

Fig. 5 Fibers near front rollers, two kinds of fibers are mixed perfectly in each cross section of blended sliver

Let these constants of the proportion be $k_{A A}$ for " $\mathrm{A}$ " and $k_{B B}$ for " $\mathrm{B}$ ". This case is equivalent to the one in which two types of sliver are placed in parallel, separately given a draft, and doubled after the front nip point. (2) Two types of fibers are blended perfectly in each cross section of blended fiber.

In this case, " $A$ " and " $B$ " as seen in Fig. 5, perfectly blend within the cross section. Unstable floating fibers of " $A$ " fibers are accelerated by the increasing velocity of the front nipped fibers of " $A$ " and " $B$ ". Let the proportional constant in the case of influence of " $\mathrm{A}$ " be $k_{A A}$ and in the case of influence of " $\mathrm{B}$ " $k_{B A} \quad$ Simplifying it gives,

$$
k_{B A}=k_{A A}=k_{A}
$$

That is, the unstable, floating fibers of " $A$ " are accelerated in proportion to the increasing velocity of all the front nipped fibers, " $A$ " and " $B$ ". The constant of the proportion in this case is $k_{A A}$.In like manner, $k_{B}$ is determined. In other words, unstable, floating fibers of " $A$ " and " $B$ " types are alternatively accelerated according to each constant $k_{A}$ and $k_{B}$ 

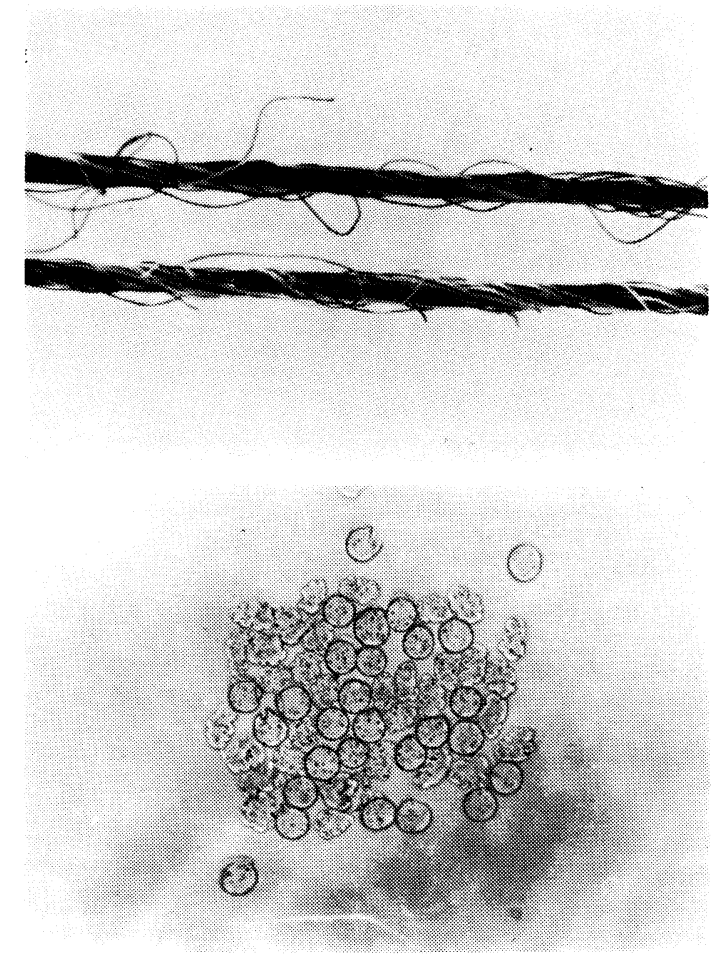

Fig. 6 An example of blended yarn polyester and rayon. fibers are mixed by blowing \& blending machine (white fiber or circular cross section fiber is poly. ester)

\section{(3) Actual case}

In the actual case, the above two basic types of mechanisms will not probably exist. Figs. 6 and 7 show two types of blended yarn, one having comparatively consistent blending, which was carried out in the blowing and blending machine and the other having inconsistent blending, which was carried out in the spinning machine by means of sliver. From these charts, it seems that the actual drafting process is situated in the intermediate stage where the above two basic structures (1) and (2). Hence, if mechanism (1) occurs with weight $(\alpha)$ and (2) with $(\beta)$, it is

$$
\alpha+\beta=1
$$

If mechanism (1) only exists

$$
\alpha=1, \beta=0
$$

If mechanism (2) only exists

$$
\alpha=0, \beta=1
$$
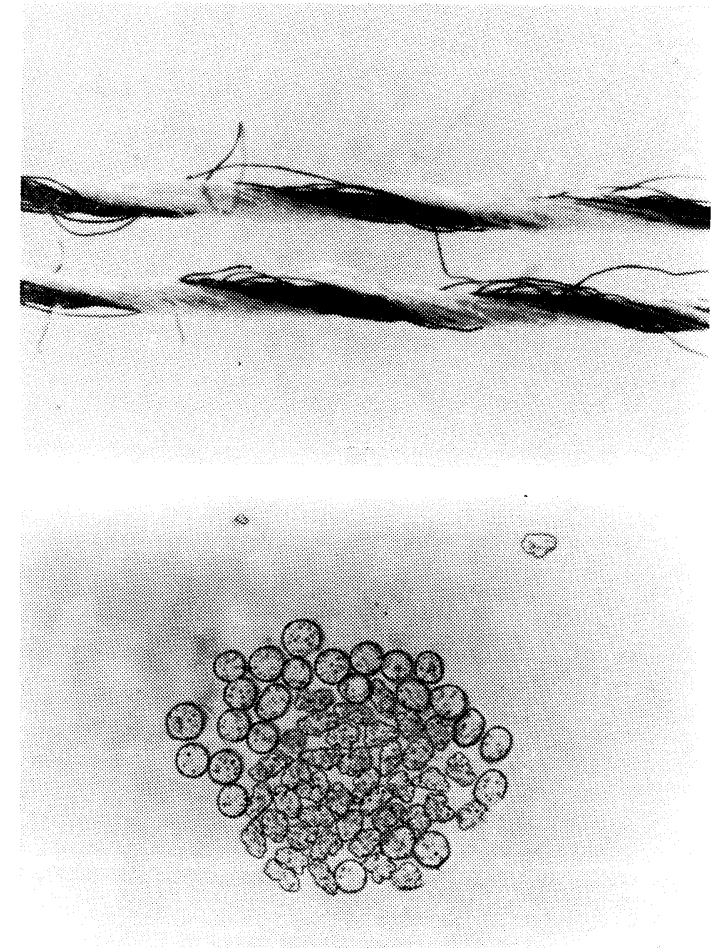

Fig. 7 An example of blended yarn. Polyester and rayon sliver are mixed by spinning machine (white fiber or circular cross section fiber is polyester)

When components composing blended yarn are not consistently blended in the cross section, or there is a too strong interaction between the same types of fiber though blending in the cross section is good, $\alpha$ may show a comparatively great value and $\beta$ a comparatively small value. As $\beta$ shows the degree of mutual interference between different fiber components, influenced mainly by blending status in the cross section, it is called "Degree of Mutual Interference".

Based on the above way of thinking, the drafting process of blended sliver was arranged as seen in Fig. 8. Generally speaking, individual fibers in blended sliver differ in friction properties, so $\mathrm{A}$ and $k_{B}$ show values different to each other. Also they differ to each other according to the draft system being capable of controlling floating fibers. If drafting conditions turn unsatisfactory, $k_{A}$ and $k_{B}$ will have greater values. With increase in $k_{A}$ and $k_{B}$ values the thickness irregularity of blended yarn becomes greater. $P_{A}=$ average blend ratio of " $A$ " fibers, so $P B(1-P A)$ is average blend ratio of " $B$ " fibers.

\section{Experimental}




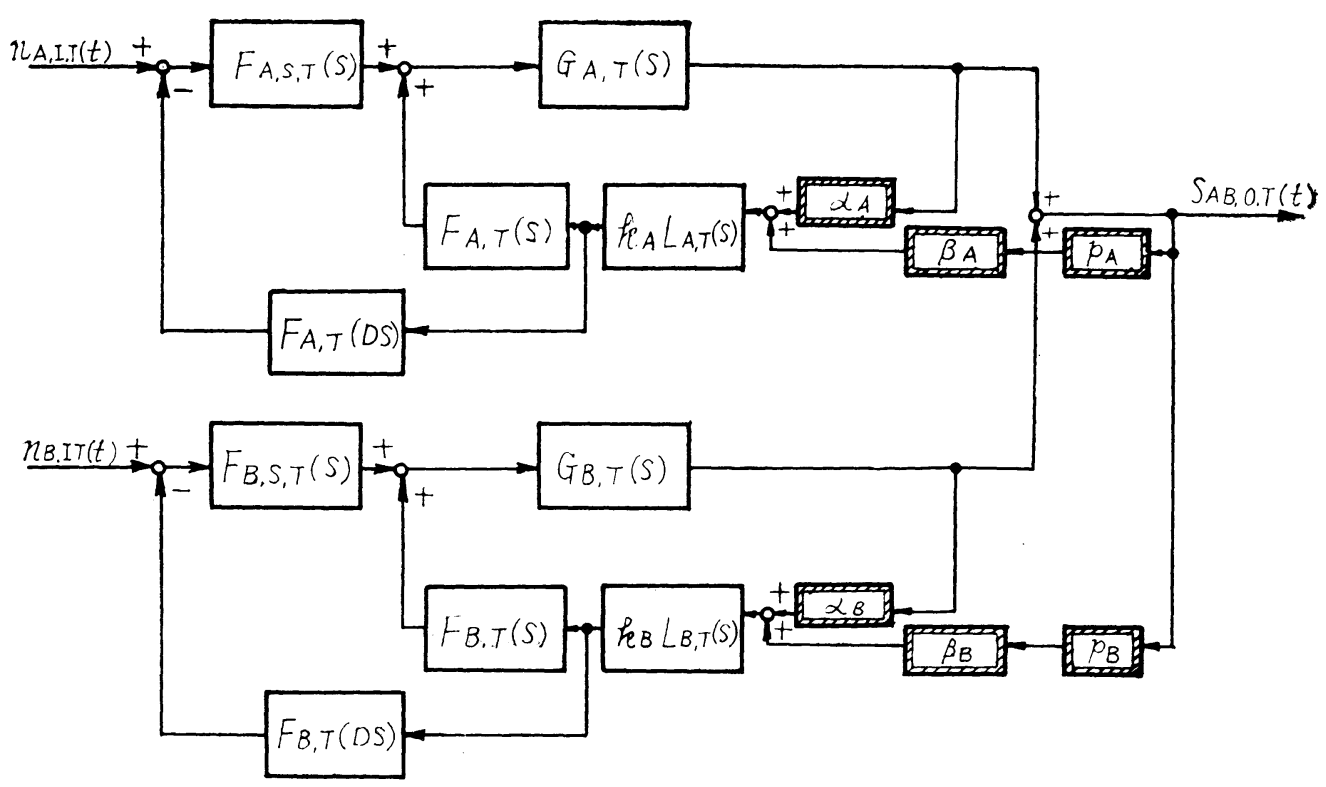

Fig. 8 Analogue block diagram of drafting process of blended sliver

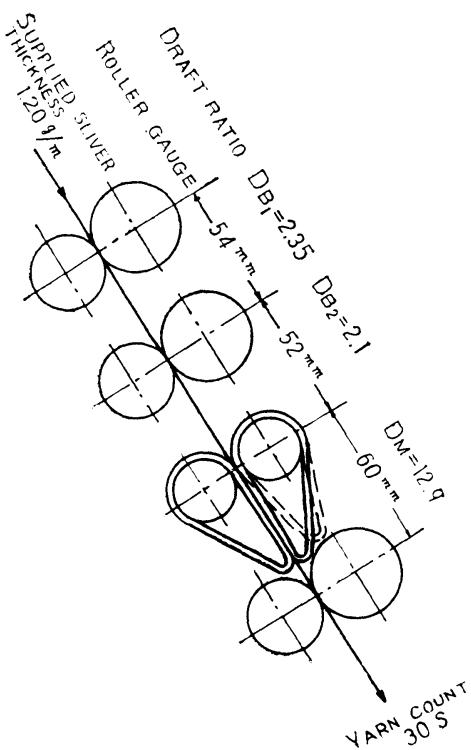

Fig. 9 Drafting conditions dotted line illustrating bad con. dition of floating fibers controlled

(2) Blending by spinning machine

As an example of non-uniform blending, polyester and rayon slivers were blended at the back nip point of a spinning frame. This is the case of $\beta$ being comparatively small in Fig. 8.

\section{3-1-3. Spinning conditions}

With respect to thickness and blend ratio irregularities occurring in the main drafting zone of a spinning frame, we made analyses. Fig. 9 shows draft ratio, roller guage and yarn count. Drafting conditions are divided into two cases.

(1) Capabilities of the draft system controlling floating fibers of each component are satisfactory. This is normal spinning conditions.

(2) Those capabilities are unsatisfactory. To clarify the character of blend ratio irregularity caused by drafting, thickness irregularity was increased by lessening the capability of the draft system with an apron the place of which is shown in Fig. 9. This case corresponds to $k_{A}$ and $k_{B}$ being greater in Fig. 8 .

3-14. Measuring techniques

In this research, one component contained in blended yarn was dissolved in a solvent while the yarn retained the fiber form, and discarded. Changes in thickness at the same part of the fiber before and after the dissolving were continuously measured along the yarn axis. Then, blend ratio irregularity was calculated.

3-14-1. Measurement of evenness of blended yarn

Evenness of yarn was measured with an electrocapacity type evenness tester having an electrode width 
of $2 \mathrm{~mm}$. In order to secure the correspondence of each cross section before and after the dissolving, marks were put on the yarn along its axis at intervals of $50 \mathrm{~cm}$.

3-14-2. Methods of dissolving

(1) Dissolving of rayon

(A) Wind up a blended yarn specimen, the evenness of which was previously measured, on a spool, about $5 \mathrm{~cm}$ diameter under little or no tension.

(B) Steep the woundup specimen in $\mathrm{H}_{2} \mathrm{SO}_{4}$ of $70 \%$ concentration, about 200 times the volume of the specimen at 22 to $25^{\circ} \mathrm{C}$, approx $15 \mathrm{~min}$. Shake it 10 to $15 \mathrm{sec}$ at intervals of $1 \mathrm{~min}$. Then, wash and dry. So that the point of contact of the specimen with the glass rod is thoroughly dissolved, repeat the above dissolving procedure by shifting the point of contact of the yarn. (C) Wash $10 \mathrm{~min}$ in running water. Neutralize with dilute ammonia (1\%), 50 times the volume of the specimen. Wash again and dry.

\section{(2) Dissolving of polyester part}

The dissolving procedure for rayon applies except the solvent and the temperature. The former is a mixed solution of 4 parts of $\mathrm{CHCl}_{2} \mathrm{CHCl}$

solution of 4 parts of $\mathrm{CHCl}_{2}$ and 6 parts of $\mathrm{C}_{6} \mathrm{H}_{5} \mathrm{OH}$ and the latter $45 \mathrm{C}$.

It was confirmed that the specimen processed according to the above procedure had one component perfectly dissolved and the other unhurt. In this experiment, for the blend containing more than average $40 \%$ polyester, rayon was dissolved, and for the less content of polyester, polyester was dissolved and discarded.

Fig. 10 illustrates an example of a thickness irregularity curve obtained before and after the dissolving. Curve (1) is the one before the dissolving and curve (2) the one after rayon part being dissolved. The thickness of the dissolved rayon is obtainable by subtracting the thickness of curve (2) from that of curve (1).

\section{3-1-5. Calculation}

At intervals of $0.5 \mathrm{~cm}$ along the yarn axis, 600 readings of thickness in the cross section were obtained from the thickness irregularity curve. and the following statistic was calculated.

(1) Coefficient of variation in the thickness of blended yarn and its two components in each cross section along the yarn axis.

(2) Coefficient of variation in the blend ratio of polyes-

ter in each cross section along the yarn axis.

(3) Correlation coefficient between the thicknesses of the two components in each cross section.

(4) Correlation coefficient between the thickness of the blended yarn and the blend ratio of polyester component

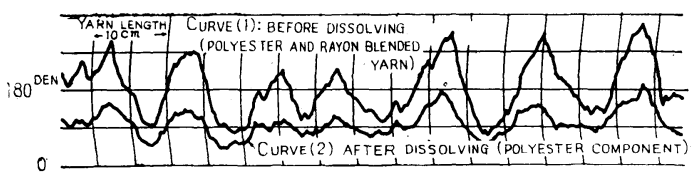

Fig. 10 An example of thickness irregularity curve before and after dissolving

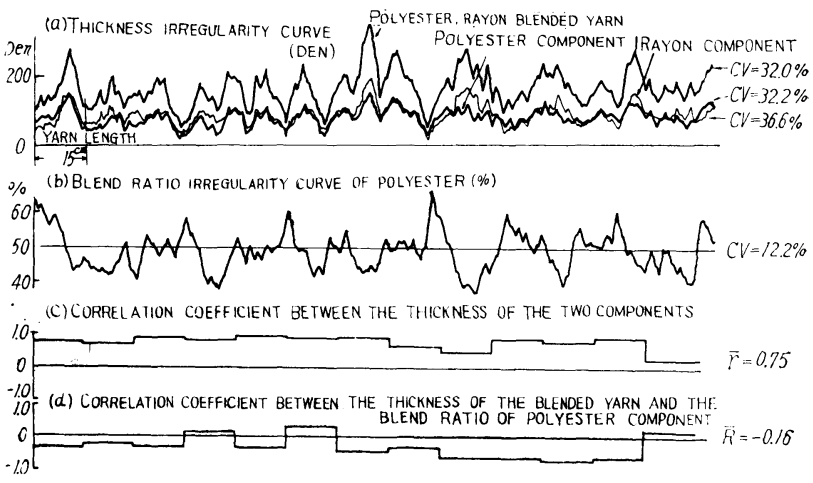

Fig. 11 Experimental results when polyester (A), rayon (A) are mixed by blowing and blending machine and are drafted under good conditions of floating fibers controlled

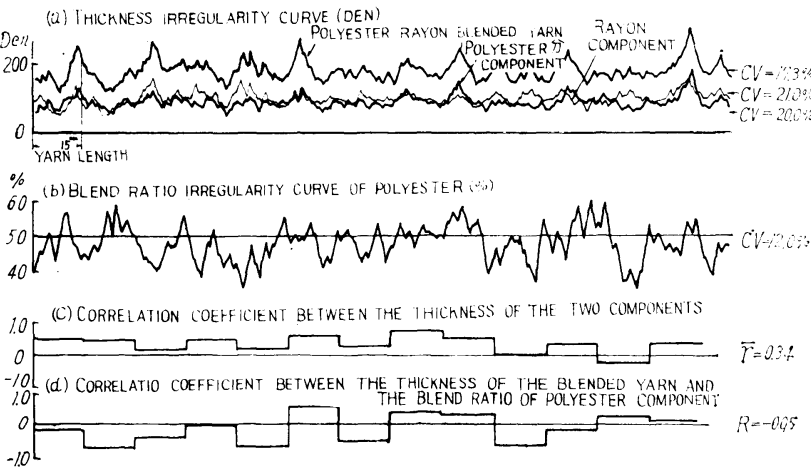

Fig. 12 Experimental results when polyester (A), rayon (A) are mixed by blowing and blending machine and draftet under bad conditions of floating fibers controlled

in each cross section. Further, each auto correlation function and spectrum density were calculated with a digital computer through the curves for thickness irregularity and blend ratio irregularity.

3-2. Experimental results and discussion 
3-2-1. Actual conditions of blend ratio irregularity

Prior to detailed analysis being made, the following fact-finding investigation of ordinary blended yarns with respect to thickness and blend ratio irregularities was carried out.

In order to check the correctness of the model drafting process in Fig. 8, an experiment with blended sliver composed of two types of fibers was carried out with respect to irregularity of thickness and per cent blend.

3-1. Procedure

3-1-1. Materials

As given in Table 1, materials are polyester fiber " $A$ " and "B", and rayon staple "A" and "B".

(1) Polyester fiber " $A$ " and rayon staple " $A$ " have an staple length of $51 \mathrm{~mm}$. Rayon is more difficult to control by the draft system than polyester (When they are separately drafted, rayon staple " $A$ " has a variation coefficient of $19.4 \%$ of thickness irregularity but polyester " $\mathrm{A}$ " has that of $15.0 \%$ ).

Table 1. Fibers Used in Experiments and Thickness Irregularity of Yarn When Each Component Was Drafted Individually

\begin{tabular}{|c|c|c|c|c|}
\hline \multirow{2}{*}{ Fiber } & \multirow{2}{*}{$\begin{array}{l}\text { Denier } \\
\text { (D) }\end{array}$} & \multirow{2}{*}{$\begin{array}{c}\text { Fiber } \\
\text { length } \\
(\mathrm{mm})\end{array}$} & \multicolumn{2}{|c|}{$\begin{array}{c}\text { Thickness irregularity } \\
\text { of the yarn }(\mathrm{CV} \%)\end{array}$} \\
\hline & & & $\begin{array}{c}\text { The yarn } \\
\text { drafted under } \\
\text { good condition }\end{array}$ & $\begin{array}{c}\text { The yarn } \\
\text { drafted under } \\
\text { bad condition }\end{array}$ \\
\hline Polyester (A) & 2.5 & 51 & 15.0 & 27.5 \\
\hline Rayon (A) & 2.0 & 51 & 19.4 & 33.0 \\
\hline Polyester (B) & 1.5 & 44 & 18.6 & 32.0 \\
\hline Rayon (B) & 1.5 & 44 & 18.4 & 29.1 \\
\hline
\end{tabular}

(2) Polyester and rayon " $B$ " are $44 \mathrm{~mm}$ in length.

Contrary to (1), polyester is hard to control as compared with rayon.

3-1-2. Methods of blending

(1) Blending by blowing and blending machine

As an example of comparatively uniform blending in the cross section of blended sliver, polyester and rayon were blended with a blowing and blending machine. This is equivalent to $\beta$ in Fig. 8 being comparatively great.

(1) Specimen: Polyester "A" and rayon " $A$ "

Rayon " $A$ " has a higher thickness irregularity than polyester " $A$ " when they are drafted separately.

(2) Blending: The place of the blending = blowing and blending machine

(3) Spinning conditions: Capability of the draft system satisfactory enough to control floating fibers of each component

(4) Average per cent blend: 50\%

Fig. 11 shows the test results. In the chart, $(a)=$ thickness irregularity curves for blended yarn and each component, $(b)=$ polyester blend ratio irregularity curve calculated from the numerical values of $(a),(c)=$ correlation coefficient between the thicknesses of each component in each cross section, calculated as $15 \mathrm{~cm}$ yarn length being a group, and $(d)=$ correlation coefficient between the thickness of blended yarn and the blend percentage of polyester in each cross section, calculated as $15 \mathrm{~cm}$ yarn length being a group.

The thickness irregularity curve of each component (Fig. $11 a$ ) shows that there are no big differences between the coefficient of variation in thickness irregularity of each component, polyester's being $20.0 \%$ and rayon's being $21.0 \%$. As seen in Fig. 11 c, the correlation coefficient between the thickness of polyester and rayon in each cross section is 0.34 as the total yarn length $(300 \mathrm{~cm})$ being a group but if $15 \mathrm{~cm}$ yarn length is a group, there are considerable variations in thickness, some parts showing a correlation coefficient of 0.5 or above and others little or no correlation. Fig. $11 \mathrm{~b}$ shows that polyester blend ratio in each cross section along the yarn axis varies from 40 to $60 \%$ against the average value of $50 \%$ and that blend ratio irregularity seems to be closely related to the evenness of the yarn.

3-2-2. Relationship between thickness and blend ratio irregularities

It seems that the trend mentioned above becomes conspicuous when thickness irregularity caused by drafting is high. With the same materials as in the previous experiment, polyester " $A$ " and rayon " $A$ " being blended in a blowing and blending machine, a blended yarn having a high thickness irregularity was spun by making unsatisfactory the capabilities of the draft system to control floating fibers of each component. This experiment corresponds to $k_{A}$ and $k_{B}$ of the model test being mode greater in Fig. 8.

Fig. 12 shows the results. The coefficient of variation in the thickness irregularity of polyester component (Fig. 12 a) is $32.2 \%$ and rayon's counterpart $36.6 \%$ (The differences are significant at the $5 \%$ level). Fig. $12 \mathrm{~d}$ shows that the correlation coefficient between the thickness of the blended yarn in each cross section and polyester content in the cross section is -0.16 (negative correlation) and that in the thick cross section polyester is comparatively less contained and more rayon is found. By comparing the thickness irregularity curve with the

Vol. 14, No. 1 (1968) 
blend ratio irregularity curve, it was found that they are closely correlated and that blend ratio irregularity occurs by drafting. For further detailed analysis, spectrum density was calculated by the value in the thickness irregularity curve (Fig. 13). The chart shows that the wavelengths of thickness irregularities of blended yarn and each component show the strongest intensity in the length about two times the staple length $(51 \mathrm{~mm})$ and that the wavelength component of blend ratio irregularity corresponds to that of thickness irregularity. These results indicate that uneven blend percentage due to drafting is closely related to thickness irregularity. Fig. 12 c shows that the correlation coefficient between thicknesses of each component in each cross section is 0.75 and that this figure is comparatively great as compared in the case of lower thickness irregularity (Fig. 11 c). Hence, it is understood that the thicknesses of two different types of fibers is strongly correlated to each other, when thickness irregularity caused by drafting is high.

These experimental results can clearly be explained by the model drafting process in Fig. 8 .

3-2-3. Relationship between fiber properties and blend ratio irregularity

The above measured results indicate that a component, which would show the higher thickness irregularity if it was spun separately, (rayon in this example) has shown the higher unevenness in the blended yarn and that the thick part of the blended fiber contains the more amounts of this component.

To assure this, with polyester " $B$ " and rayon " $B$ " having contrary properties to polyester " $A$ " and rayon " $A$ " (When drafted individually, polyester " $B$ " has a higher thickness irregularity than rayon "B"), a blended yarn was produced, the blending taking place in the blowing and blending machine (average blend percentage of 50\%) and the draft system being not in good conditions to control floating fibers of each component. The results are shown in Fig. 14. The coefficient of variation in the thickness irregularity of polyester component is $41.7 \%$ and that of rayon $36.9 \%$. The polyester component shows a higher unevenness (The differences are significant at the 5\% level). The correlation coefficient between the thickness of blended fiber in each cross section and the polyester content is 0.18 . This shows that in the thick part of the blended yarn, the polyester component, which would show a higher thickness irregularity when drafted separately from the other component, is contained in comparatively larger quantities. This is true even in the case of the good control of floating fibers

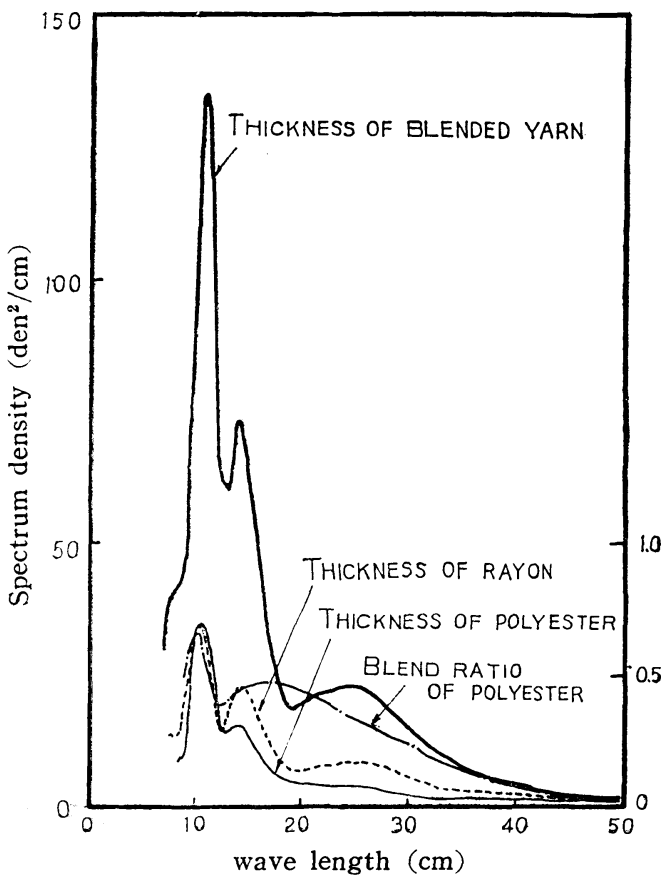

Fig. 13 Spectrum density of thickness irregularity and blend ratio irregularity

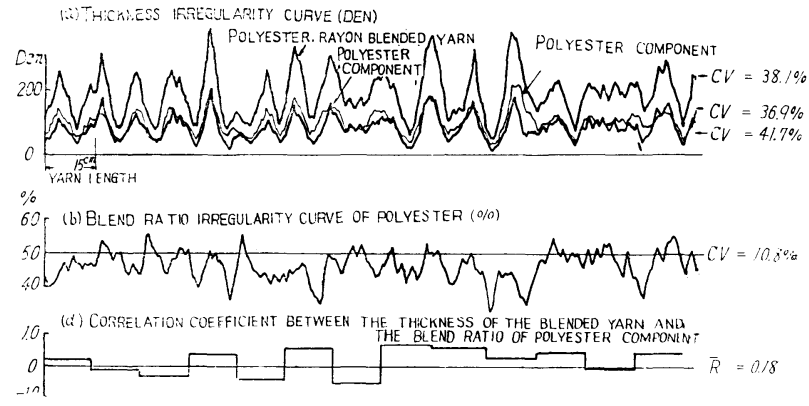

Fig. 14 Experimental results when polyester (B), rayon (B) are mixed by blowing and blending machine and are drafted under bad condition of floating fibers controlled

by the draft system, though its chart was omitted. Through the above experiment, it was confirmed that the thickness irregularity in blended yarn of each component quantitatively has a trend similar to that caused when each component is spun individually.

3-2-4. Relationship between average blend ratio and blend irregularity

Polyester " $A$ " and rayon " $A$ " were blended in a blowing and blending machine with varying average blend ratios, and with the draft systems of satisfactory and 
unsatisfactory controls of floating fibers of each component, a blended yarn was spun. Fig. 15 shows the coefficient of variations in thickness irregularity of blended fiber and each component. Generally, the coefficient of variation in thickness irregularity of rayon is higher than that of polyester component. This trend is the same as in 50\% average blend ratio (Fig. 11). As average blend ratio decreases, the coefficient of variation in the thickness irregularity of that component increases. However, the component does not much influence the mechanical properties of the blended yarn, since the average blend ratio is small.

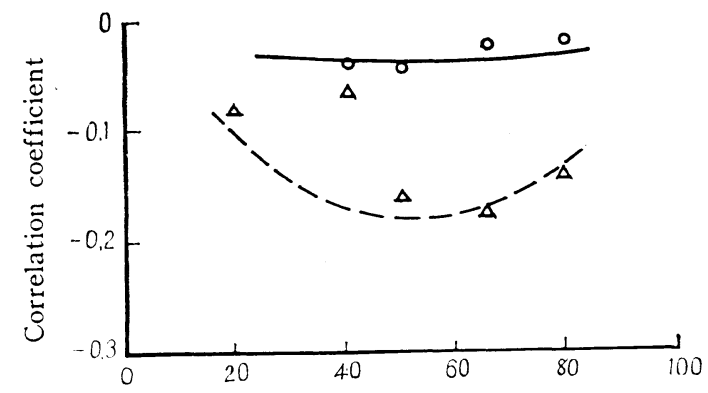

Average blend ratio of polyester (\%)

Fig. 16 The correlation coefficient between the thickness of the blended yarn and the blend ratio of polyester component in each cross section

- illustrating the yarn drafted under good condition of floating fiber controlled

.... illustrating the yarn drafted under bad condition

Fig. 16 shows the correlation coefficient between the thicknesses of blended yarn and polyester content in the cross section. These values are all negative and so in the thick part of the blended yarn much rayon is contained. Fig. 17 shows the correlation coefficient in thicknesses of each component in each cross section when average blend ratio is varied. When average blend ratio is $50 \%$, the correlation coefficient is highest.

Fig. 18 shows the spectrum density of thickness irregularity of blended yarn and each component when blend ratio is varied. The wavelengths of the thickness irregularity of blended yarn and each component in any blend ratio agree with each other. The yarn having a comparatively high thickness irregularity possesses spectrum density with high kurtosis in the wavelength about two times the yarn length and the yarn having a low thickness irregularity possesses fairly high spectrum density in the same wavelength.

3-2-5. Relationship between blend uniformity in the cross section and blend ratio irregularity

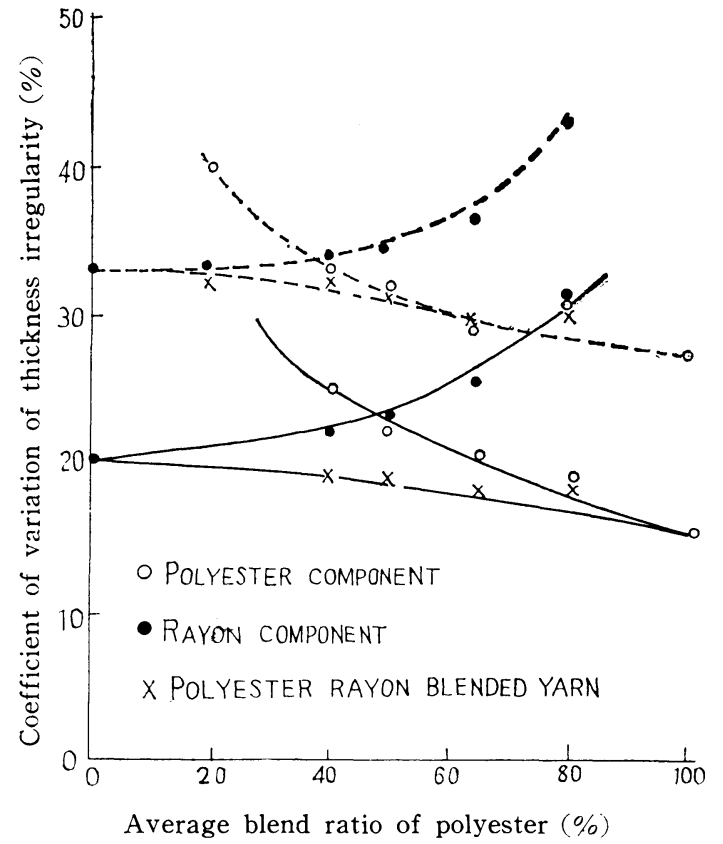

Fig. 15 The thickness irregularity of blended yarn and its two components at various average blend ratio

- illustrating the yarn drafted under good condition of floating fiber controlled

-. - illustrating the yarn drafted under bad conditions

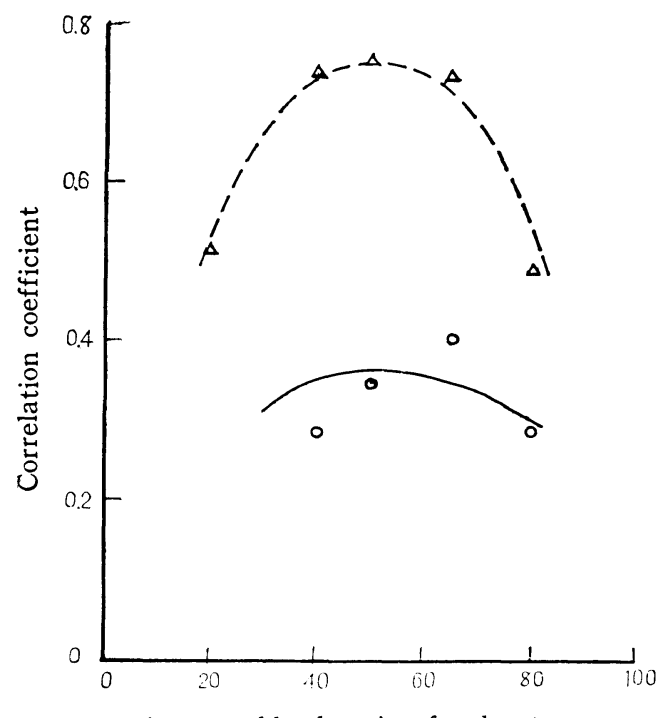

Average blend ratio of polyester

Fig. 17 The correlation coefficient between the thickness of the two components in each cross section

- illustrating the yarn drafted under good condition of floating fiber controlled

... illustrating the yarn drafted under bad condition 
It is estimated that blend uniformity in the cross section of two types of fibers affects the mutual motion of the two types of fibers in the drafting zone and consequently affects blend ratio irregularity. To affirm: this estimate, the yarn uniformly blended in the cross section, the two component fibers of which are blended in the blowing and blending machine was compared with the yarn non-uniformly blended in the cross section, the two components of which were blended in the spinning frame, with respect to thickness and blend ratio irregularities. This experiment is equal to parameters $(\alpha)$ and $(\beta)$ being changed in the model process of Fig. 8. Fig. 19 shows the measured results of blended yarn with 50\% average blend ratio, spun in an unsatisfactory condition in which floating fibers of each component are difficult to control, after polyester " $\mathrm{A}$ " and rayon " $\mathrm{A}$ " have been mixed in sliver form on a spinning frame. The yarn blended on a spinning frame (Fig. 19) is clearly contrasted with the yarn blended in a blowing and blending machine in that each component of the former shows thickness irregularity in conditions almost independent of each other. As seen in Table 2, this phenomenon is shown by the correlation coefficient between the thickness of the polyester part and that of the rayon part in the blended yarn. In the case of the blending on a spinning frame, the correlation coefficient is smaller as compared with the blending in the blowing and blending machine. Blend ratio irregularity, however, is higher in the case of the blending in a spinning frame by the reason indicated in Fig. 3. The inference that non-uniform blending in the cross section of blended sliver weakens the mutual interference of motion between two types of fibers in the drafting zone, resulting in blend ratio irregularity getting higher, was affirmed by these experimental results. In other words, these results are self-explanatory by the model drafting process in Fig. 8 .

The fact that the correlation coefficient in the thickness of each component of blended yarn shows some values except $O$, even when each component is separate at the back nip point of a spinning frame in the case of the spinning frame blending, indicates that two types of sliver are being grabbed at the front nip point in the form of tight doubling, so the drafting behaviors of each sliver start an interaction to some extent-not perfectly independent of each other.

3-2-6. Relationship between fiber length of each component of blended yarn and blend ratio irregularity

The polyester and rayon components combined in the previous experiments had the same staple length but in

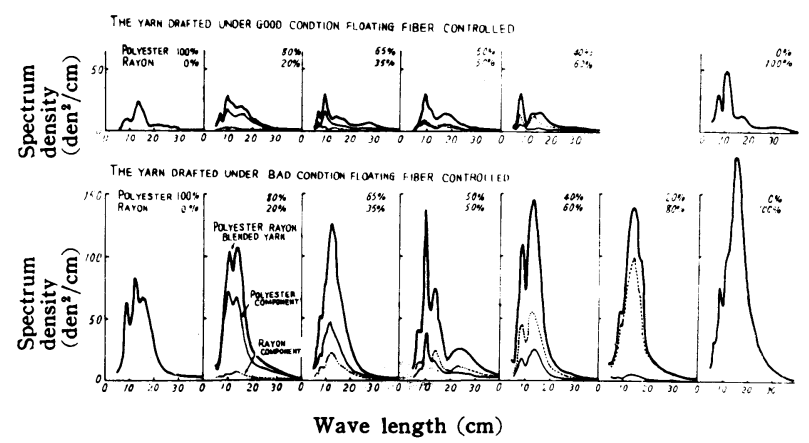

Fig. 18 Spectrum density of thickness irregularity of various yarns

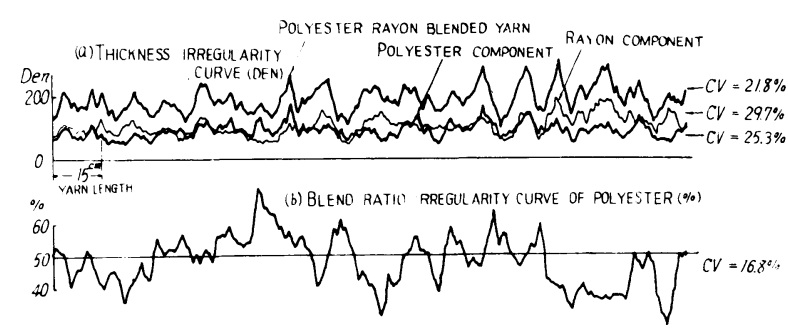

Fig. 19 Experimental results when polyester (A), rayon (A) are mixed by spinning machine and are drafted under bad condition of floating fibers controlled

Table 2. Correlation Coefficient in Thickness of Each Component Composing Blended Yarn (Average blend ratio : 50\%)

\begin{tabular}{|c|c|c|c|c|c|}
\hline \multicolumn{2}{|c|}{ Fiber } & \multicolumn{2}{|c|}{ Polyester (A) } & \multicolumn{2}{|c|}{ Polyester (B) } \\
\hline \multicolumn{2}{|c|}{$\begin{array}{l}\text { Drafting condition } \\
\text { of floating } \\
\text { fiber controlled }\end{array}$} & Good & $\mathrm{Bad}$ & Good & $\mathrm{Bad}$ \\
\hline \multirow[t]{2}{*}{ Mixing } & $\begin{array}{l}\text { Flowing } \\
\text { and } \\
\text { blending } \\
\text { machine }\end{array}$ & 0.34 & 0.75 & 0.45 & 0.85 \\
\hline & $\begin{array}{l}\text { Spining } \\
\text { Machine }\end{array}$ & 0.05 & 0.25 & 0.20 & 0.33 \\
\hline
\end{tabular}

this experimentation, we used different staple lengths of polyester and rayon. It is conceivable that if the draft system is unsatisfactory to control floating fibers of each component when two types of fibers having different staple lengths are to be blended, the component with the shorter staple length has more unstable, floating fibers than the component with the longer staple length, result- 
Table 3. Coefficient of Variation in Thickness Irregularity of Each Component of Blended Yarn (in the case of Diffierent Staple Lengths of Polyester and Rayon)

\begin{tabular}{c|c|c|c}
\hline \multirow{2}{*}{ Fibers } & \multirow{2}{*}{$\begin{array}{c}\text { Drafting condition } \\
\text { of floating } \\
\text { fiber controlled }\end{array}$} & \multicolumn{2}{|c}{$\begin{array}{c}\text { CV of thickness o } \\
\text { of each componant }\end{array}$} \\
\cline { 3 - 4 } & Polyester & Rayon \\
\hline Polyester A $(51 \mathrm{~mm})$ & Good & 18.0 & 18.0 \\
\hline Rayon B $(44 \mathrm{~mm})$ & Bad & 29.9 & 33.1 \\
\hline Polyester B $(44 \mathrm{~mm})$ & Good & 21.7 & 19.3 \\
\hline Rayon A $(51 \mathrm{~mm})$ & Bad & 36.8 & 28.9 \\
\hline
\end{tabular}

Polyester A, $51 \mathrm{~mm}$ Rayon B, $44 \mathrm{~mm}$

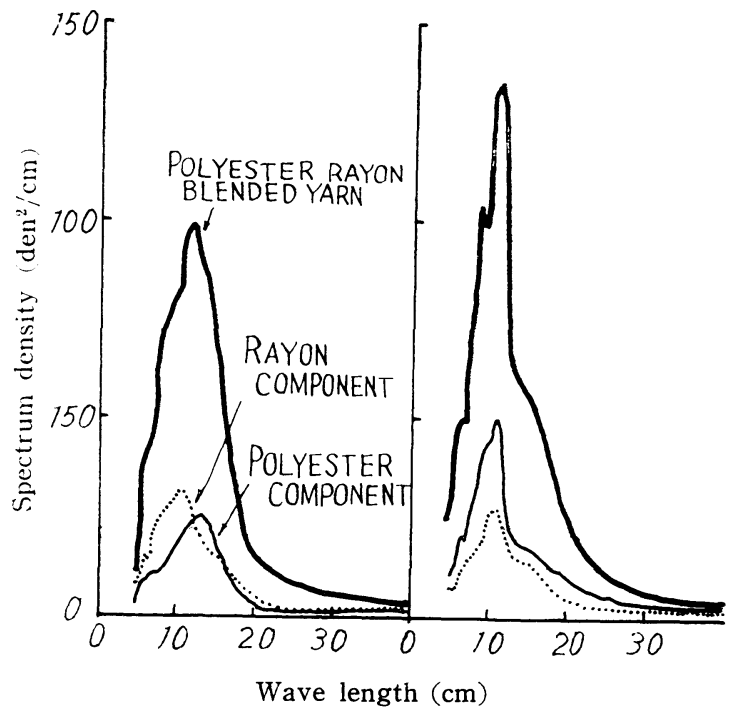

Fig. 20 Spectrum density of thickness irregularity

ing in high thickness irregularity. To make sure this estimate, two types of blended yarn were experimentally produced, one, polyester " $A$ ", $51 \mathrm{~mm}$ long and rayon " $\mathrm{B}$ ", $44 \mathrm{~mm}$ long and the other, polyester " $B$ " $44 \mathrm{~mm}$ long and rayon " $A$ ", $51 \mathrm{~mm}$ long. Table 3 , shows the coefficient of variation in the thickness of each component. The component with shorter staple length shows a higher thickness irregularity than that with longer staple length.

Fig. 20 shows the spectrum density of the thickness irregularity of blended yarn. In the case of blended yarn by the combinations of polyester " $A$ " and rayon " $B$ " which are comparatively easy to control by the draft system, the spectrum density of the thickness irregulari- ty of each component presents the separate kurtosis in the wavelength about two times each slaple length. In the case of the combination of polyester " $B$ " and rayon " $A$ ", which are hard to control by the draft system, rayon " $A$ " is influenced by polyester " $B$ ", the thickness of which easily becomes uneven and the peak of the wavelength which should correspond to the staple length of rayon " $A$ " shifts to the position of the peak of polyester " $B$ ".

\section{Conclusion}

As analogue model shown in Fig. 8 is proposed to explain the mechanism of the blend ratio irregularity caused by drafting. The experimental results show that the model is suitable to describe the mechanism. We could get the following results using this model and experimental data.

(1) The blend ratio irregularity much occurs during drafting. Strong correlation is observed between the yarn thickness irregularity curve and blend ratio irregularity curve and the same drafting wave as is seen in the drafted yarn thickness is observed on the blend ratio irregularity curve, and both have the very similar wave length spectrum.

(2) The thicker cross section of the yarn contains relatively larger number of fibers of one component of which the floating fibers are more difficult to control than the other component.

(3) When these two components are mixed perfectly in the cross section, the thickness irregularity of each component has strong correlation and the blend ratio irregularity is relatively small.

However, when these two components are located separately in the cross section of yarn, this correlation is weaker but the blend ratio irregularity becomes larger.

\section{References}

[1] M. Hannah; J. Text. Inst., 44, T436 (1953)

[2] G. V. Lund; Text Res. J., 24, 759 (1954)

[3] M. J. Coplan, W. G. Klein; Text. Res. J., 25, 743 (1955)

[4] M. J. Coplan, W. G. Klein; Text. Res. J., 26, 914 (1956)

[5] D. R. Cox; J. Text. Inst., 45, T113 (1954)

[6] M. J. Coplan, M. G. Bloch; Text. Res. J., 25, 902 (1955)

[7] M. J. Coplan, W. G. Klein; Text Res. J., 29, 632 (1959)

[8] K. Fujino, S. Kawabata; J. Text Mach. Soc. Japan, Englished, 1962, 8 3, 